\title{
Thermodynamics of oxygen defective Magnéli phases in rutile: A first-principles study
}

\author{
Leandro Liborio* \\ Department of Chemistry, Imperial College London, London SW7 2AZ, United Kingdom \\ Nicholas Harrison \\ Department of Chemistry, Imperial College London, London SW7 2AZ, United Kingdom \\ and STFC, Daresbury Laboratory, Daresbury, Warrington WA4 4AD, United Kingdom
}

(Received 23 October 2007; revised manuscript received 16 January 2008; published 7 March 2008)

\begin{abstract}
$A b$ initio thermodynamics has been used to calculate the formation energies, in different environmental conditions, for a number of oxygen-defective structures in rutile. In addition to the $\operatorname{Ti}_{n} \mathrm{O}_{2 n-1}(3 \leqslant n \leqslant 5)$ Magnéli phases, the two fundamental points defects, Ti interstitials and neutral oxygen vacancies, were also considered. The predicted phase stability is compared to available experimental data and there is reasonable agreement between the calculated phase boundaries and those observed. These results are used to discuss a mechanism that has been proposed as an explanation for the formation of the crystallographic shear planes in rutile.
\end{abstract}

DOI: $10.1103 /$ PhysRevB.77.104104

PACS number(s): 91.60.Ed, 47.50.Cd, 61.46.-w

\section{INTRODUCTION}

The titanium-oxygen system plays an important role in the study of nonstoichiometry. Rutile, one of the most studied phases of $\mathrm{TiO}_{2-x}$, only exists in nonstoichiometric form ${ }^{1}$ and has a complicated defect structure. ${ }^{2,3}$ For low $x$, the point defects that dominate are oxygen vacancies and titanium interstitials. $^{4-6}$ As the sample is reduced, defect structures with long range order form: The so-called Magnéli phases. ${ }^{3,7}$ $\mathrm{X}$-ray diffraction measurements reveal that these phases are extended planar defect structures, ${ }^{2,7,8}$ the crystallographic shear planes $(\mathrm{CS})$.

The chemistry of point defects in $\mathrm{TiO}_{2-x}$ has been analyzed previously in terms of Schottky and Frenkel defects as well as oxygen vacancies and titanium interstitials. ${ }^{9-12}$ Although very rare, titanium vacancies ${ }^{13}$ have also been studied. Several experimental ${ }^{13-17}$ and theoretical ${ }^{4-6,12}$ investigations have been performed in order to understand the role that point defects play in determining the physical and chemical properties of $\mathrm{TiO}_{2-x}$. Results of particular relevance to the current study will be discussed in Secs. (5) and (6).

$\mathrm{TiO}_{2-x}$ has many practical applications: For example, it is used as a photocatalyst, ${ }^{18}$ a gas sensor, a white pigment, and an optical coating. ${ }^{19}$ In all of these applications the Magnéli phases are likely to be present and may influence important properties. In many cases the effects are poorly understood. There are also particular technological applications that exploit the substoichiometry such as electrochemistry ${ }^{20}$ and tribology. ${ }^{21}$

The defective nature of the oxide has profound consequences for the structure and electronic structure that are revealed, in part, by electronic measurements. Bartholomew and Frankl ${ }^{22}$ measured the electrical conductivity of $\mathrm{Ti}_{n} \mathrm{O}_{2 n-1}$ with $3 \leqslant n \leqslant 6$ within the temperature range $78-298 \mathrm{~K}$. Inglis et $a .^{23}$ studied the temperature dependence of the electrical conductance of $\mathrm{Ti}_{n} \mathrm{O}_{2 n-1}$ with $4 \leqslant n \leqslant 9$ in the $4-320 \mathrm{~K}$ temperature range. In these studies no single conduction mechanism was found to dominate across the series. In particular, for $\mathrm{Ti}_{4} \mathrm{O}_{7}$, both works reported discontinuities in the conductivity as the temperature was changed: A semiconductor-semiconductor transition in the $125-140 \mathrm{~K}$ temperature range and a semiconductor-metal transition at about $150 \mathrm{~K}$.

$\mathrm{Ti}_{4} \mathrm{O}_{7}$ is the Magnéli phase where the bulk of the experimental analysis had been focused. Marezio et al. ${ }^{24}$ performed single-crystal $\mathrm{x}$-ray investigations of the charge localization in $\mathrm{Ti}_{4} \mathrm{O}_{7}$. These investigations revealed a charge localization in the insulating phases of $\mathrm{Ti}_{4} \mathrm{O}_{7}$ in which chains of alternating $\mathrm{Ti}^{+3}$ and $\mathrm{Ti}^{+4}$ ions run parallel to the pseudorutile $c$ axis. The $\mathrm{Ti}^{+3}$ ions are ordered in alternate $\mathrm{Ti}^{+3}-\mathrm{Ti}^{+3}$ pairs up to $150 \mathrm{~K}$ above which temperature the $\mathrm{Ti}^{+3}-\mathrm{Ti}^{+3}$ pairs separate and all equivalent titanium ions adopt an average electronic charge- $\mathrm{Ti}^{+3.5}$ Abbatte et al. $^{25}$

Lakkis et al. ${ }^{26}$ combined x-ray diffraction with electron paramagnetic resonance studies. They proposed a mechanism for the metal-insulator transition related to the disorder of the $\mathrm{Ti}^{+3}$ pairs. Abbatte et al. ${ }^{25}$ presented results of photoemission spectroscopy and x-ray absorption spectra (XAS) within the $50-300 \mathrm{~K}$ range. They studied $\mathrm{Ti}_{4} \mathrm{O}_{7}$ 's electronic structure across the semiconductor-semiconductor and semiconductor-metal transitions. They observed sudden changes in the $\mathrm{O} 1 \mathrm{~s}$ XAS at the semiconductor-metal transition, and a $0.25 \mathrm{eV}$ upward shift of the Ti $3 d$ bands in the low temperature semiconducting phase. The behavior of the electronic states at the phase transitions has also been analyzed by Kobashi et al. ${ }^{27}$ Using photoemission spectroscopy, they studied the electronic structure and measured $\mathrm{Ti}_{4} \mathrm{O}_{7}$ 's band gap. The optical properties of $\mathrm{Ti}_{4} \mathrm{O}_{7}$ have been experimentally investigated as well. Watanabe et al. ${ }^{28}$ measured $\mathrm{Ti}_{4} \mathrm{O}_{7}$ 's reflection spectra as a function of temperature. They found it changed discontinuously at about 130 and $150 \mathrm{~K}$, the electronic phase transition temperatures. Leonov et al. ${ }^{29}$ performed, to the best of the authors' knowledge, the only $a b$ initio study on $\mathrm{Ti}_{4} \mathrm{O}_{7}$. Using the local density approximation (LDA) $+U$ method, they investigated $\mathrm{Ti}_{4} \mathrm{O}_{7}$ 's charge ordering and found a charge-ordered insulating solution with an energy gap of $0.29 \mathrm{eV}$.

The magnetic properties of these phases have also been studied experimentally. $\mathrm{Ti}_{n} \mathrm{O}_{2 n-1}$ with $3 \leqslant n \leqslant 6$, in their pure 
phases, present semiconductor-metal transitions that were studied by measuring the magnetic susceptibility. ${ }^{30}$ In particular, for $\mathrm{Ti}_{4} \mathrm{O}_{7}$, together with the semiconductor-metal transition that takes place at around $150 \mathrm{~K}$, there is a strong increase in the paramagnetic susceptibility. At temperatures below $150 \mathrm{~K}, \mathrm{Ti}_{4} \mathrm{O}_{7}$ is antiferromagnetic. ${ }^{30,31}$

The subject of the current work is the thermodynamic stability of defect phases, which has been measured in a series of experimental studies. In their seminal paper on higher titanium oxides, Bursill and $\mathrm{Hyde}^{3}$ performed an analysis of the thermodynamics of the Magnéli phases with $4 \leqslant n \leqslant 10$. Their work explored the coexistence, at equilibrium, of Magnéli phases with closely related structures, for instance, $\mathrm{Ti}_{4} \mathrm{O}_{7}$ and $\mathrm{Ti}_{5} \mathrm{O}_{9}$. More recently, Walder and Eriksson ${ }^{1}$ presented a complete study of the titanium-oxygen system, starting from metallic titanium and continuing up to the oxygen deficient $\mathrm{TiO}_{2-x}$, including the Magnéli phases. They constructed a $T-x$ phase diagram, with $T$ ranging from $298 \mathrm{~K}$ up to around $2000 \mathrm{~K}$, at $1 \mathrm{~atm}$.

\section{THEORY}

The thermodynamic formalism presented here is designed to compare the relative stability of different oxygendefective rutile structures. The computed thermodynamics is based consistently on the results of total energy electronic structure calculations. This methodology is closely related to that which has been used extensively to compare the free energy of surfaces with different stoichiometries. ${ }^{32,33}$ This section will focus on the application of this formalism to bulk oxygen-defective structures, in particular, the Magnéli phases.

It is assumed that the systems under study are in equilibrium. Essentially, the relative stability of the oxygendefective structures will be analyzed through the Gibbs formation energy of the oxygen defects. The physical picture is that of a system initially composed of pure bulk rutile, in which neutral oxygen vacancies are created. Therefore, in its final equilibrium state, the system will have either a bulk rutile structure, with oxygen point defects, titanium interstitials, or the Magnéli phase structure, with oxygen planar defects. Each of these alternative structures will be in equilibrium with bulk rutile and with an oxygen reference gas phase. Once equilibrium has been reached, there will be three phases in the system: The solid Magnéli phase $\mathrm{T}_{n} \mathrm{O}_{2 n-1}$ (or the bulk solid with point defects), the bulk $\mathrm{TiO}_{2}$ phase, and the reference gas $\mathrm{O}_{2}$ phase. It is a three phase system with three components and therefore it has two degrees of freedom, which are chosen here to be $T$ and $p_{\mathrm{O}_{2}}$.

The Gibbs formation energy of an oxygen defect at $T$ and $p_{\mathrm{O}_{2}}, \Delta G_{f}\left(T, p_{\mathrm{O}_{2}}\right)$, is

$$
\begin{aligned}
\Delta G_{f}\left(T, p_{\mathrm{O}_{2}}\right)= & \frac{1}{n_{\mathrm{TiO}_{2}}}\left[G^{\text {supercell }}\left(T, p_{\mathrm{O}_{2}}\right)+n_{\mathrm{O}}^{\text {def }} \mu_{\mathrm{O}}^{\text {ref }}\left(T, p_{\mathrm{O}_{2}}\right)\right. \\
& \left.-G_{\mathrm{TiO}_{2}}^{\text {bulk }}\left(T, p_{\mathrm{O}_{2}}\right)\right],
\end{aligned}
$$

where $G^{\text {supercell }}\left(T, p_{\mathrm{O}_{2}}\right)$ is the Gibbs free energy of a rutile's defective supercell with $n_{\mathrm{TiO}_{2}}$ units of $\mathrm{TiO}_{2} \cdot G_{\mathrm{TiO}_{2}}^{\text {bulk }}\left(T, p_{\mathrm{O}_{2}}\right)$ indicates the Gibbs free energy of an equivalent nondefective rutile's supercell. For a system with $n_{\mathrm{TiO}}$ units of $\mathrm{TiO}_{2}$, $G_{\mathrm{TiO}_{2}}^{\text {bulk }}\left(T, p_{\mathrm{O}_{2}}\right)$ can be written as $n_{\mathrm{TiO}_{2}} \mu_{\mathrm{TiO}_{2}}^{\text {bulk }}\left(T, p_{\mathrm{O}_{2}}\right)$. $n_{\mathrm{O}}^{\text {def }}$ indicates the number of oxygen defects in the defective supercell and $\mu_{\mathrm{O}}^{\mathrm{ref}}\left(T, p_{\mathrm{O}_{2}}\right)$ is the oxygen chemical potential at $T$ and $p_{\mathrm{O}_{2}}$.

Equation (1) is a general expression and can be applied to different oxygen defective configurations in rutile. For instance, applying Eq. (1) to a Magnéli phase results in

$$
\begin{aligned}
\Delta G_{f}^{\mathrm{Ti}_{n} \mathrm{O}_{2 n-1}}\left(T, p_{\mathrm{O}_{2}}\right)= & \frac{1}{n_{\mathrm{TiO}_{2}}}\left[G_{\mathrm{Ti}_{n} \mathrm{O}_{2 n-1}}^{\text {supercell }}\left(T, p_{\mathrm{O}_{2}}\right)+n_{\mathrm{O}}^{\text {def }} \mu_{\mathrm{O}}^{\text {ref }}\left(T, p_{\mathrm{O}_{2}}\right)\right. \\
& \left.-n_{\mathrm{TiO}_{2}} \mu_{\mathrm{TiO}_{2}}^{\text {bulk }}\left(T, p_{\mathrm{O}_{2}}\right)\right]
\end{aligned}
$$

whereas, applying Eq. (1) to a rutile supercell with one isolated oxygen defect gives

$$
\begin{aligned}
\Delta G_{f}^{\mathrm{def}}\left(T, p_{\mathrm{O}_{2}}\right)= & \frac{1}{n_{\mathrm{TiO}_{2}}}\left[G_{\mathrm{def}}^{\text {supercell }}\left(T, p_{\mathrm{O}_{2}}\right)+\mu_{\mathrm{O}}^{\mathrm{ref}}\left(T, p_{\mathrm{O}_{2}}\right)\right. \\
& \left.-n_{\mathrm{TiO}_{2}} \mu_{\mathrm{TiO}_{2}}^{\text {bulk }}\left(T, p_{\mathrm{O}_{2}}\right)\right] .
\end{aligned}
$$

Equations (3) and (2) are formally equivalent. The key difference between them lies in $G^{\text {supercell }}\left(T, p_{\mathrm{O}_{2}}\right)$ : It is possible to have either isolated oxygen point defects $\left[G_{\text {def }}^{\text {supercell }}\left(T, p_{\mathrm{O}_{2}}\right)\right]$ or interacting planar defects $\left[G_{\mathrm{Ti}_{n} \mathrm{O}_{2 n-1}}^{\text {supercell }}\left(T, p_{\mathrm{O}_{2}}\right)\right]$, as in a Magnéli phase.

These formation energies are normalized to the number of $\mathrm{TiO}_{2}$ bulk units. To be explicit, for any specific $T$ and $p_{\mathrm{O}_{2}}$, if the normalized Gibbs formation energy of an oxygen defect in a Magnéli phase is smaller than in a point-defective slab of rutile, the Magnéli phase is more stable than the point defective slab, i.e., it is thermodynamically favorable for the oxygen defect to form in a Magnéli phase.

The temperature and pressure dependence of the free energies and chemical potentials of all condensed phases in Eqs. (3) and (2) were neglected. For all condensed phases the free energy is therefore approximated by the total energy at the athermal limit. Taking the Magnéli phase as an example, within this approximation, Equation (2) may be rewritten as Eq. (4)

$$
\begin{aligned}
\Delta G_{f}^{\mathrm{Ti}_{n} \mathrm{O}_{2 n-1}}\left(T, p_{\mathrm{O}_{2}}\right)= & \frac{1}{n_{\mathrm{TiO}_{2}}}\left[E_{\mathrm{Ti}_{n} \mathrm{O}_{2 n-1}}^{\text {supercell }}(0 \mathrm{~K})-n_{\mathrm{TiO}_{2}} E_{\mathrm{TiO}_{2}}^{\text {bulk }}(0 \mathrm{~K})\right. \\
& \left.+n_{\mathrm{O}}^{\text {def }} \mu_{\mathrm{O}}^{\mathrm{ref}}\left(T, p_{\mathrm{O}_{2}}\right)\right] .
\end{aligned}
$$

The approximations used for transforming Eq. (2) into Eq. (4) have been described in detail elsewhere, for instance, in Refs. 33 and 34 This methodology has also been used in the past to study the reconstructions of GaAs surfaces ${ }^{35,36}$ and defects in $\mathrm{ZnO}^{37}$

It is clear from Eq. (4) that the remaining dependence of $\Delta G_{f}^{\mathrm{Ti}_{n} \mathrm{O}_{2 n-1}}\left(T, p_{\mathrm{O}_{2}}\right)$ on $p_{\mathrm{O}_{2}}$ and $T$ is through the chemical potential or gaseous oxygen, $\mu_{\mathrm{O}}^{\text {ref }}\left(T, p_{\mathrm{O}_{2}}\right)$. To an excellent approximation the gas can be considered to be an ideal gas and thus it is possible to put bounds on the physical values of $\mu_{\mathrm{O}}^{\mathrm{ref}}\left(T, p_{\mathrm{O}_{2}}\right)$ and express $\Delta G_{f}^{\mathrm{Ti}_{n} \mathrm{O}_{2 n-1}}\left(T, p_{\mathrm{O}_{2}}\right)$ as a function of $\mu_{\mathrm{O}}^{\text {ref }}$ only. 
Within this approximation the variation of $\mu_{\mathrm{O}}^{\mathrm{ref}}\left(T, p_{\mathrm{O}_{2}}\right)$ with $T$ and $p_{\mathrm{O}_{2}}$ is 32,34

$$
\begin{aligned}
\mu_{\mathrm{O}_{2}}^{\text {ref }}\left(p_{\mathrm{O}_{2}}, T\right)= & E_{0}+\left(\mu_{\mathrm{O}_{2}}^{0}-E_{0}\right)\left(\frac{T}{T^{0}}\right)-C_{p} T \ln \left(\frac{T}{T^{0}}\right) \\
& +k T \ln \left(\frac{p_{\mathrm{O}_{2}}}{p_{\mathrm{O}_{2}}^{0}}\right) .
\end{aligned}
$$

The value of $\mu_{\mathrm{O}_{2}}^{0}$ determines the reference energy for the chemical potential of the gas and must be consistent with the reference for the bulk calculations. It can be calculated at standard temperature and pressure from the experimental formation Gibbs free energy of a series of oxides ${ }^{38}$ and the calculated free energies of their condensed phase components. The standard chemical potential of oxygen used in this work was calculated as the average of those obtained from the Gibbs energies of formation of the oxides: $\mathrm{TiO}_{2}$ rutile and anatase, $\mathrm{Ti}_{4} \mathrm{O}_{7}$ and $\mathrm{Ti}_{3} \mathrm{O}_{5}$.

In principle, Eq. (5) is defined for any value of $\mu_{\mathrm{O}_{2}}^{\text {ref }}\left(p_{\mathrm{O}_{2}}, T\right)$ and therefore limits on the allowable range that are consistent with the equilibrium of all possible phases must be imposed. For instance, if $\mu_{\mathrm{O}_{2}}^{\text {ref }}\left(p_{\mathrm{O}_{2}}, T\right)$ becomes too low, all oxygen would leave the sample, i.e., the $\mathrm{TiO}_{2}$ oxide would decompose into Ti metal and oxygen gas. The analytical formulation of the previous statements allows one to defined limits on $\mu_{\mathrm{O}}^{\mathrm{ref}}\left(p_{\mathrm{O}_{2}}, T\right)$ for which the supercell is stable with respect to decomposition.

As it is assumed that the slab is in equilibrium with bulk $\mathrm{TiO}_{2}$, the chemical potentials of $\mathrm{Ti}$ and $\mathrm{O}$ in the supercell must satisfy

$$
\mu_{\mathrm{TiO}}^{\text {bulk }}=\mu_{\mathrm{Ti}}^{\text {supercell }}+2 \mu_{\mathrm{O}}^{\text {ref }}\left(p_{\mathrm{O}_{2}}, T\right)
$$

and the titanium chemical potential in the supercell must be less or equal to the one in the bulk phase

$$
\mu_{\mathrm{Ti}}^{\text {slab }} \leqslant \mu_{\mathrm{Ti}}^{\text {bulk }}
$$

by combining Eqs. (6) and (7) we get

$$
\mu_{\mathrm{O}}^{\mathrm{ref}}\left(p_{\mathrm{O}_{2}}, T\right) \geqslant \frac{\mu_{\mathrm{TiO}_{2}}^{\text {bulk }}-\mu_{\mathrm{Ti}}^{\text {bulk }}}{2},
$$

which is a lower bound for $\mu_{\mathrm{O}}^{\mathrm{ref}}\left(p_{\mathrm{O}_{2}}, T\right)$. The lower bound is a hard bound: $\mu_{\mathrm{O}}^{\mathrm{ref}}\left(p_{\mathrm{O}_{2}}, T\right)$ cannot go below it without the bulk crystal decomposing. In principle, it is possible to find an upper bound for $\mu_{\mathrm{O}}^{\mathrm{ref}}\left(p_{\mathrm{O}_{2}}, T\right)$. The bound would be in oxygen-rich conditions, at the point beyond which oxygen would start to condense on the sample. But, in the temperature and pressure range of interest, an oxygen condensed solid phase does not exist. Therefore, similar to Eq. (7), an appropriate estimate of the upper limit of $\mu_{\mathrm{O}}^{\text {ref }}\left(p_{\mathrm{O}_{2}}, T\right)$ is the internal energy of the gas extrapolated to the athermal limit which is half the total energy of the oxygen molecule at $0 \mathrm{~K}$, $E_{0} / 2$.

\section{MAGNÉLI PHASES}

The titanium-oxygen system has long been important in the study of nonstoichiometry. ${ }^{1,3}$ The higher oxides in this system are distinguished from the lower by an internal structural consistency, which strongly influences their properties.

The Magnéli phases $\operatorname{Ti}_{n} \mathrm{O}_{2 n-1}$, with $4 \leqslant n \leqslant 9$, may be generated from the rutile structure by the operation $\frac{1}{2}[0 \overline{1} 1]$ $\times(121)_{r}{ }^{2,7}$

The Magnéli phases' structure may be described as being built up of $\mathrm{TiO}_{6}$ octahedra joined by edges and corners to form slabs of rutile-type structure. The slabs' thickness is $n \mathrm{TiO}_{6}$ octahedra and they extend parallel to the $(121)_{r}$ planes, with adjacent slabs mutually displaced by $\frac{1}{2}[0 \overline{1} 1]_{r}$. Since, in the tetragonal rutile structure, $\frac{1}{2}[0 \overline{1} 1]_{r}$ is precisely an oxygen-oxygen vector, the net of oxygens is continuous across the $(121)_{r}$ planes, but the Ti nets are in antiphase, ${ }^{3}$ i.e., the filled and empty rows of octahedral interstices are interchanged across each (121) $r$ boundary. This is why the (121) planes are known as crystallographic shear planes (CS plane). ${ }^{2}$ In the real Magnéli structures the ideal network of oxygen atoms changes slightly after a $\frac{1}{2}[0 \overline{1} 1]_{r}$ displacement. The oxygen-oxygen distances around the CS plane are smaller than in bulk rutile. ${ }^{2}$

$\mathrm{TiO}_{6}$ octahedra at the $(121)_{r}$ boundary share faces, which makes the atomic arrangement in the boundary region similar to that present in the corundum-type structure adopted by $\mathrm{Ti}_{2} \mathrm{O}_{3}$.

Figure 1(a) shows a view along the $[100]_{r}$, of only one anion+cation layer of rutile (oxygens are the gray circles and titaniums are the small black circles). In both figures a small topological distortion has been introduced which flattens the puckered oxygen planes of rutile resulting in an oxygen packaging that may be idealized to perfect h.c.p. This flattened model was proposed by Bursill and Hyde, ${ }^{3}$ and it helps in the visualization of rutile and its derived structures. The rows of titaniums, filling the octahedral interstices in Fig. 1(a), are the titaniums of the edge-shared $\mathrm{TiO}_{6}$ octahedra in rutile. These octahedra are parallel to the rutile $c$ axis. In the anion+cation layers of rutile's bulk structure, the pattern of filled and empty titanium sites is identical for all layers, with adjacent layers being displaced relatively by $\frac{1}{2}[1 \overline{1} 1]_{r}$.

As previously mentioned, the formal geometrical operation $(121) \frac{1}{2}[0 \overline{1} 1]_{r}$, applied to pure rutile, produces a CS plane. If this operation is repeated, for instance, on one in every 8 (121) anion planes, the $\mathrm{Ti}_{4} \mathrm{O}_{7}$ structure is obtained. Figure 1(b) shows a (100) cation+anion layer of $\mathrm{Ti}_{4} \mathrm{O}_{7}$. It can be seen that the strings of interstitials titaniums, along the rutile $c$ axis, are folded back on themselves in a reverse $Z$ shape. Each one of these $Z$ 's introduces one face-shared pair of $\mathrm{TiO}_{6}$ octahedra parallel to the [100] direction in rutile. Moreover, each one of these Z's can be thought of as made up of an antiphase boundary plus an extra titanium interstitial.

\section{COMPUTATIONAL METHOD}

Total energy calculations were performed using the CASTEP code, ${ }^{39}$ which implements density functional theory within the plane-wave pseudopotential approximation. Ultrasoft pseudopotentials were used. ${ }^{40}$ In these calcu- 


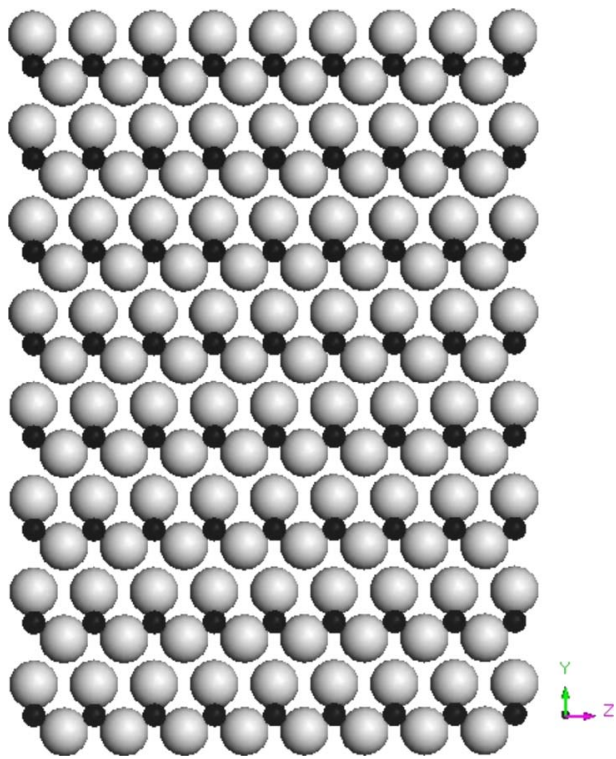

(a)

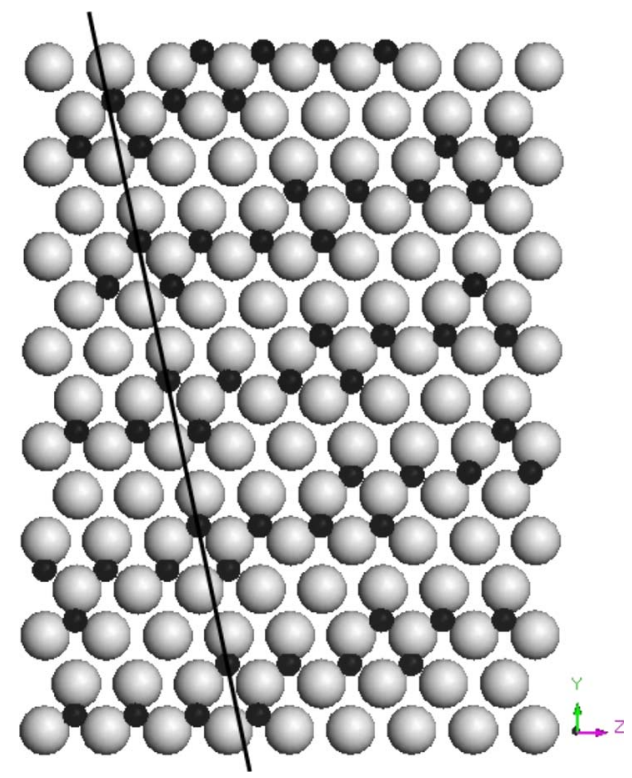

(b)
FIG. 1. (Color online) Panel (a) is a [100] view of a cation + anion layer of idealized rutile. Panel (b) shows a [100] projection of a cation+anion layer in the $\mathrm{Ti}_{4} \mathrm{O}_{7}$ Magnéli phase. The small black circles are titanium. The black line in (b) indicates where the $(121)_{r}$ plane intersects this plane of atoms. lations, electronic exchange and correlation were described using the local density approximation (LDA). The sampling of $k$ space was performed on a Monkhorst-pack grid and the density of points was determined from a spacing of $0.041 / \AA$ in all the Magnéli phases, and using a $(2 \times 2 \times 2)$ Monkhorst-Pack grid for the point defect calculations. A plane-wave cutoff of $460 \mathrm{eV}$ was used in both cases.

\section{A. Point defects}

Two elemental point defects of oxygen defective rutile were studied in this paper: The neutral oxygen vacancy and the titanium interstitial. Oxygen atoms in rutile are equivalent by symmetry, but the position of the interstitial cation needs to be considered more carefully. Rutile has open channels in the $0,1 / 2, z$ direction, with the $[1 / 2,1 / 2,1 / 2]$ position being the centre of an octahedron of oxygen atoms. It has been suggested that this position is the most stable for location of the titanium interstitial. ${ }^{19,41}$ This is a reasonable assumption, since the environment for the titanium interstitial in this site is closer to that of Ti in bulk rutile. Different starting positions along the $z$ axis were tested, and it was confirmed that the octahedral sites are the most stable positions for the titanium interstitials.

A primitive unit cell containing two formula units was used as a model for stoichiometric rutile. The calculated crystal parameters of this unit cell were $a=b=4.573 \AA$ and $c=2.943 \AA$. These are in good agreement with experimental values: $a=b=4.586 \AA$ and $c=2.954 \AA .{ }^{42}$

To calculate the formation energy of the point defects, a supercell approach was used. As the aim was to investigate isolated defects in an infinite solid, the lattice constant of the supercell was constrained to be that of the nondefective bulk rutile, and only internal coordinates were relaxed. ${ }^{37}$ The convergence limit for the forces on the atoms was $0.01 \mathrm{eV} / \AA$. Within this force tolerance, a supercell size of $(3 \times 3 \times 3)$ was sufficient to converge the defect formation energies to within $0.001 \mathrm{eV}$ per formula unit.
The experimentally measured oxygen vacancy concentration in rutile, at $1100{ }^{\circ} \mathrm{C}$ and near atmospheric pressure, is $0.5 \% .^{15}$ In the present study, the vacancy concentration was $0.9 \%$. Lower concentrations would have implied larger supercells, which were computationally very expensive to simulate.

\section{B. Magnéli phases}

Initial structural data for the relaxations of $\mathrm{Ti}_{4} \mathrm{O}_{7}$ and $\mathrm{Ti}_{5} \mathrm{O}_{9}$ were taken from Andersson and Jahnberg. ${ }^{7}$ For $\mathrm{Ti}_{3} \mathrm{O}_{5}$, the data were taken from Asbrink and Magnéli. ${ }^{43}$ The force tolerance for the structural relaxation was also $0.05 \mathrm{eV} / \AA$, which again was sufficient to converge the defect formation energies to within $0.001 \mathrm{eV}$ per formula unit.

\section{RESULTS}

\section{A. Point defects}

Numerous experimental studies have been carried out on the nonstoichiometry of rutile as a function of oxygen partial pressure. Among others, these studies include thermogravimetry ${ }^{44,45}$ and electrical conductivity measurements. ${ }^{14,45,46}$ Although the types of major defects in rutile are still under discussion, the studies generally conclude that $\mathrm{TiO}_{2-x}$ is oxygen deficient and that the predominant atomic point defects are interstitial titanium ions and/or oxygen vacancies.

With regard to previous theoretical results, to the authors knowledge there have been no $a b$ initio studies of the stability of point defects in rutile as a function of the oxygen partial pressure and temperature.

Catlow and James ${ }^{47}$ used interatomic potentials to calculate the formation energies of point and extended defects. For nearly stoichiometric $\mathrm{TiO}_{2-x}$, they predicted anion vacancies $\left(\mathrm{O}^{-2}\right)$ to be the predominant point defects. At higher defects concentrations, these anion vacancies were predicted to ag- 

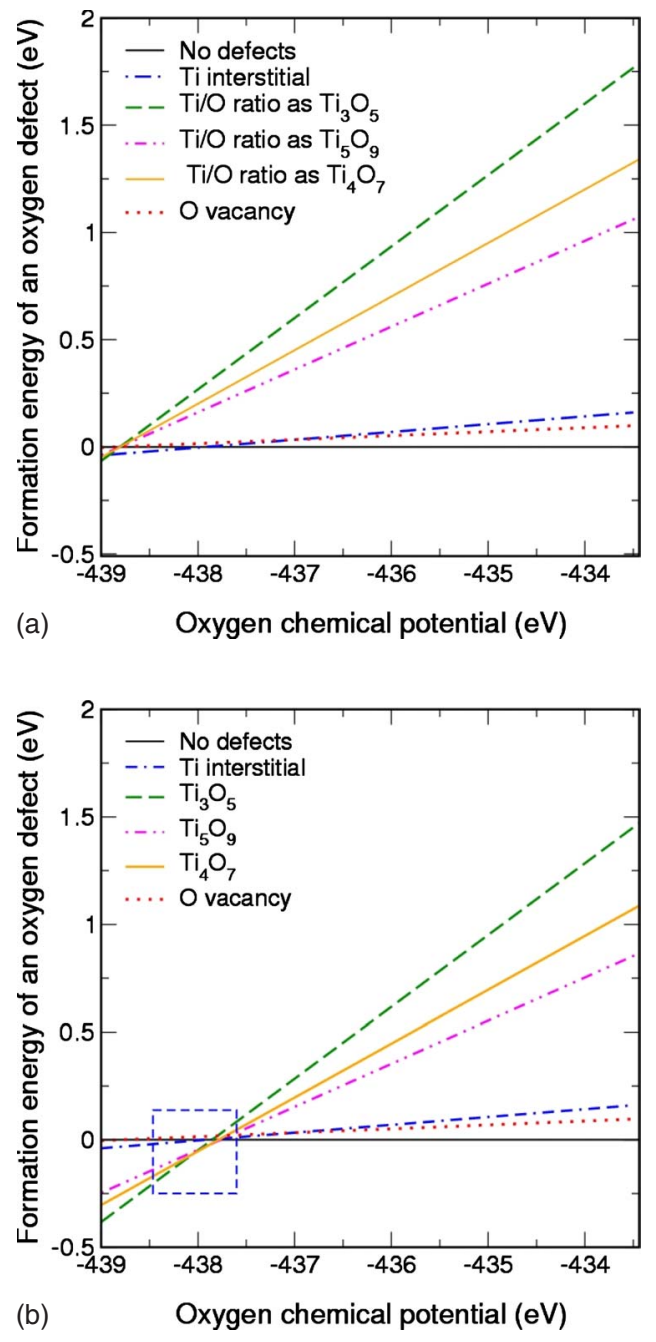

FIG. 2. (Color online) Defect formation energies $\left[\Delta G_{f}^{\mathrm{def}}\left(T, p_{\mathrm{O}_{2}}\right)\right.$ and $\left.\Delta G_{f}^{\mathrm{Ti}_{n} \mathrm{O}_{2 n-1}}\left(T, p_{\mathrm{O}_{2}}\right)\right]$, as functions of the oxygen chemical potential $\left[\mu_{\mathrm{O}}^{\mathrm{ref}}\left(T, p_{\mathrm{O}_{2}}\right)\right]$, in oxygen-defective rutile. Panel (a) represents systems of noninteracting oxygen defects where the $\mathrm{O} / \mathrm{Ti}$ ratio is equivalent to the one in $\mathrm{Ti}_{3} \mathrm{O}_{5}, \mathrm{Ti}_{4} \mathrm{O}_{7}$, and $\mathrm{Ti}_{5} \mathrm{O}_{9}$. Panel (b) represents the $\mathrm{Ti}_{3} \mathrm{O}_{5}, \mathrm{Ti}_{4} \mathrm{O}_{7}$, and $\mathrm{Ti}_{5} \mathrm{O}_{9}$ Magnéli phases. As the oxygen vacancy and titanium interstitial are single defects, their formation energy in (a) and (b) are the same.

gregate into shear planes, i.e., the Magnéli phases.

Mazharul et al.,${ }^{5}$ Dawson et al., ${ }^{4}$ and Cho et al. ${ }^{6}$ calculated the formation energies of isolated point defects in bulk rutile, using ab initio calculations, as

$$
\begin{aligned}
\Delta G_{f}^{\text {def }}\left(T, p_{\mathrm{O}_{2}}\right)= & \Delta G_{f}^{\text {def }}(0 \mathrm{~K}) \\
= & \frac{1}{n_{\mathrm{TiO}_{2}}}\left[E_{\text {def }}^{\text {supercell }}(0 \mathrm{~K})-n_{\mathrm{TiO}_{2}} E_{\mathrm{TiO}_{2}}^{\text {bulk }}(0 \mathrm{~K})\right. \\
& \left.+n_{\mathrm{O}}^{\text {def }} E_{\mathrm{O}}(0 \mathrm{~K})\right],
\end{aligned}
$$

where $E_{\mathrm{O}}(0 \mathrm{~K})$ is half of the ground-state energy of the oxygen molecule. No temperature or oxygen partial pressure dependence has been considered here. Clearly, this formalism cannot account for the whole range of titanium-oxygen

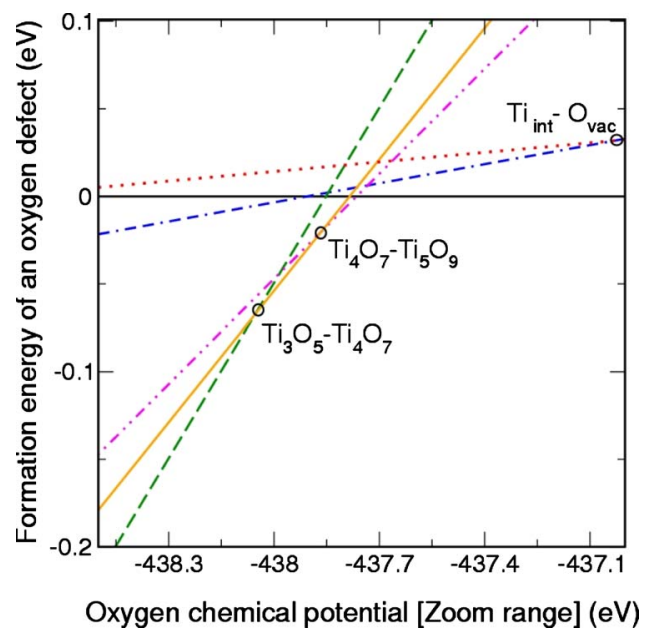

FIG. 3. (Color online) Zoom of the area highlighted in the dashed box (blue) in Fig. 2(b).

stable phases that may exist, because their stability depends on the environmental conditions, namely the oxygen partial pressure and temperature. Only Cho et al. ${ }^{6}$ estimated the lower limit of $\mu_{\mathrm{O}_{2}}^{\text {ref }}$ using the oxygen molecule's experimental dissociation energy. In this lower limit-corresponding to a titanium-rich environment- the formation energy of an oxygen vacancy equals the formation energy of a titanium interstitial. But the relative stability was seen to be reversed under titanium-poor conditions, i.e., $\mu_{\mathrm{O}}^{\mathrm{ref}}=E_{\mathrm{O}}(0 \mathrm{~K})$.

The structural relaxation effects for the oxygen vacancy and titanium interstitial are characterized here by measuring the changes of distances of the relaxed atoms from the vacancy and titanium interstitial site. Removal of the oxygen atom causes the surrounding titaniums-placed in the (110) plane-to relax outwards by $0.28-0.31 \AA$ (that is, away from the vacancy site). This is reasonable as the removal of the oxygen ion reduces the screening of the repulsion between the positively charged titanium ions. The relaxation is also in reasonable agreement with the results computed by Cho et al. ${ }^{6}$ and Mazharul et al. ${ }^{5}$ In Cho et al. ${ }^{6}$ titanium atoms were displaced by $0.27-0.30 \AA$ outward, whereas Mazharul et $a l^{5}$ observed a relaxation of $0.15-0.17 \AA$. For the case of the titanium interstitial, its two closest titanium neighbors are displaced $0.28 \AA$ outward. For the oxygens of the octahedral site, the ones in the octahedral basal plane are pulled in toward the cation interstitial by $0.21 \AA$, while the apical oxygens are relaxed outward by $0.27 \AA$. These displacement values are in good agreement with those reported by Cho et al. ${ }^{6}$

In Figs. 2(a) and 2(b) the formation energies for the titanium interstitial and the oxygen vacancy, as a function of $\mu_{\mathrm{O}_{2}}^{\text {ref }}$, can be observed. The lines representing these formation energies are included in both figures for comparison.

At low $\mu_{O}^{\text {ref }}$, rutile with interstitionally dissolved titanium is predicted to be stable, which agrees with the experimental evidence. Titanium dioxide, in its thermodynamically stable form rutile, shows nonstoichiometry in a narrow composition range at low concentration of oxygen defects. ${ }^{1}$ 

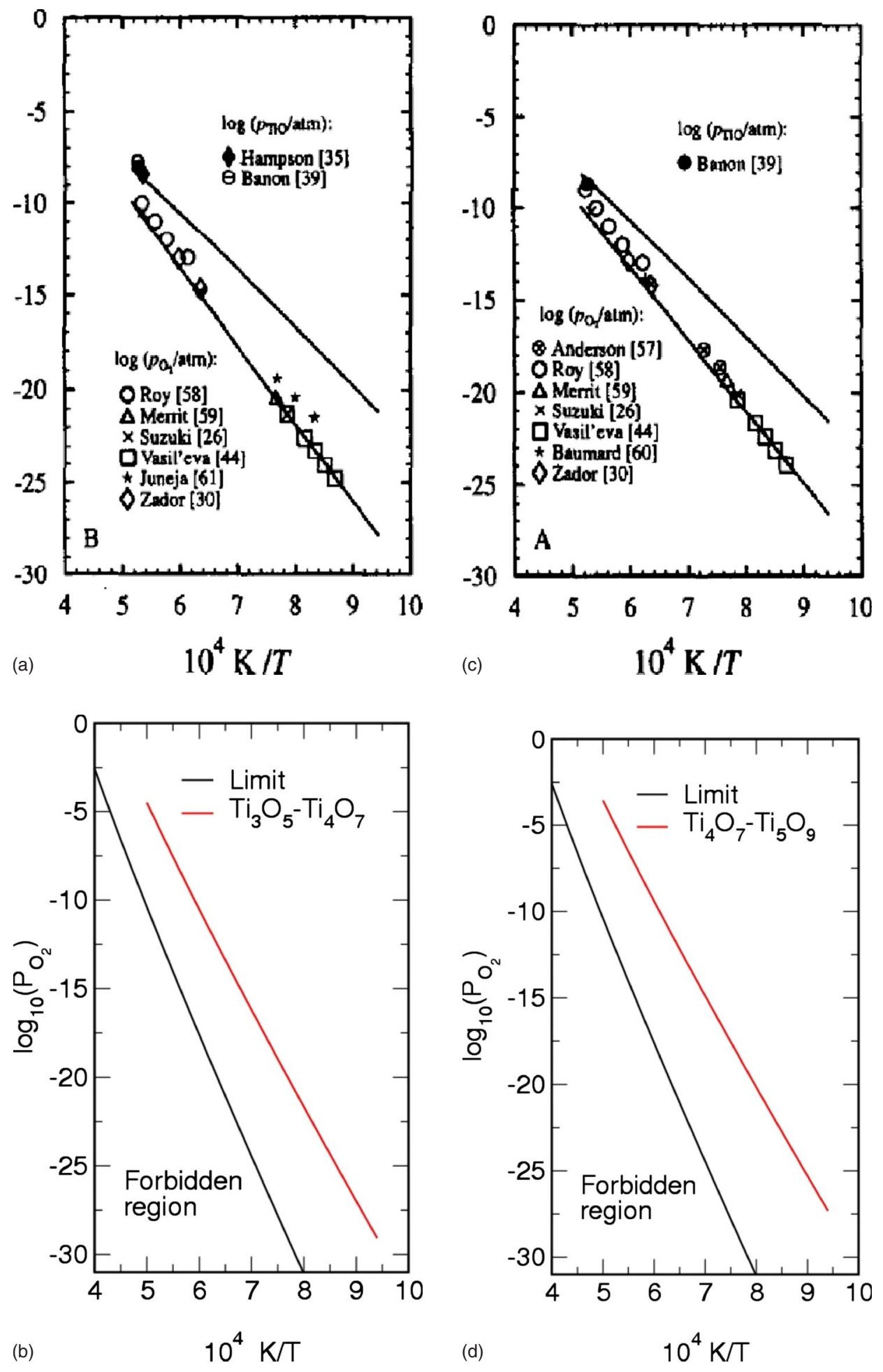

FIG. 4. (Color online) (a) and (c) are reproduced with permission from Waldner and Eriksson (Ref. 1) and show the observed oxygen partial pressuretemperature relationship. The $y$ axis shows the logarithm of the oxygen partial pressure $\left(p_{\mathrm{O}_{2}}\right)$ and the $x$ axis shows $10^{4} \mathrm{~K}$ divided by the temperature in $\mathrm{K}$. (b) and (d) are the results from the $a b$ initio thermodynamics computed here. The boundary of the thermodynamically forbidden region (black line) in these plots was calculated using the lower limit for the oxygen chemical potential.

(b)

$$
10^{4} \mathrm{~K} / \mathrm{T}
$$

\section{B. Magnéli phases}

The formation energies of isolated defects reported above are a prerequisite for the study of rutile with different concentrations of noninteracting oxygen defects. Within the formalism proposed in these paper, in a supercell with $n_{\mathrm{TiO}_{2}}$ $\mathrm{TiO}_{2}$ units, the formation energy for $n_{\mathrm{O}}^{\text {def }}$ oxygen isolated defects is

$$
\begin{aligned}
& \Delta G_{f}^{\text {def }}\left(T, p_{\mathrm{O}_{2}}\right) \\
& =\frac{n_{\mathrm{O}}^{\mathrm{def}}}{n_{\mathrm{TiO}_{2}}} \underbrace{\left[E_{\mathrm{def}}^{\text {supercell }}(0 \mathrm{~K})-n_{\mathrm{TiO}_{2}} E_{\mathrm{TiO}_{2}}^{\text {bulk }}(0 \mathrm{~K})+\mu_{\mathrm{O}}^{\mathrm{ref}}\left(T, p_{\mathrm{O}_{2}}\right)\right.}_{\Delta G_{f}^{\text {def }} \text { for one isolated defect }}]
\end{aligned}
$$

This formulation allows one to ask the following 
TABLE I. Comparison between experimental and theoretical equilibrium temperatures. The chosen oxygen partial pressure is 1 $\times 10^{-15}$ atm.

\begin{tabular}{lcclcc}
\hline \hline & \multicolumn{2}{c}{$\mathrm{Ti}_{3} \mathrm{O}_{5}-\mathrm{Ti}_{4} \mathrm{O}_{7}$} & & \multicolumn{2}{c}{$\mathrm{Ti}_{4} \mathrm{O}_{7}-\mathrm{Ti}_{5} \mathrm{O}_{9}$} \\
\cline { 2 - 3 } \cline { 5 - 6 } & $\log \left(P_{\mathrm{O}_{2}}\right)$ & $T(\mathrm{~K})$ & & $\log \left(P_{\mathrm{O}_{2}}\right)$ & $T(\mathrm{~K})$ \\
\hline Expt. & -15 & 1567.4 & & -15 & 1538.5 \\
Theor. & -15 & 1438.8 & & -15 & 1515.2 \\
\hline \hline
\end{tabular}

question: Given a particular concentration of oxygen defects, would the defects prefer to be isolated or to form a Magnéli phase?

Together with the point defects' formation energies, Figs. 2(a) and 2(b) include the energy of defective rutile with an oxygen defect concentration equivalent to $\mathrm{Ti}_{3} \mathrm{O}_{5}, \mathrm{Ti}_{4} \mathrm{O}_{7}$, and $\mathrm{Ti}_{5} \mathrm{O}_{9}$. Figure 2(a) corresponds to isolated (i.e., noninteracting) defects and Fig. 2(b) to defects in a Magnéli phase. If the defects are assumed to be noninteracting, their formation energies for all possible values of $\mu_{\mathrm{O}_{2}}^{\text {ref }}$ are positive in almost all the available range of $\mu_{\mathrm{O}_{2}}^{\text {ref }}$. On the contrary, if equivalent concentrations of oxygen defects are forming as Magnéli phases, their formation energies are systematically lower than isolated defects and, at low $\mu_{\mathrm{O}_{2}}^{\text {ref }}$, negative. This indicates that oxygen defects in rutile prefer to aggregate to form Magnéli phases as observed. ${ }^{1}$ The region of interest in Fig. 2(b) has been enclosed in a blue box. Figure 3 displays this region on a larger scale, and it is possible to appreciate in more detail the behavior of the Magnéli phases'. As the oxygen chemical potential is decreased, the structure of the material changes from one Magnéli phase to the next in order to accommodate the larger number of defects. This is the trend that has been observed in X-ray diffraction experiments. ${ }^{1,3,48}$

The crossing points between formation energies, labeled as $\mathrm{Ti}_{3} \mathrm{O}_{5}-\mathrm{Ti}_{4} \mathrm{O}_{7}$ and $\mathrm{Ti}_{4} \mathrm{O}_{7}-\mathrm{Ti}_{5} \mathrm{O}_{9}$, indicate equilibrium between two consecutive Magnéli phases. These points are associated with a particular value of the oxygen chemical potential and thus to a particular relationships between the oxygen partial pressure and the temperature which defines the equilibrium phase boundary. Using the formalism defined here (equation (5)) an analytic expression for the phase boundary can be found and compared directly to that observed.

In Fig. 4 the dependence of $\log \left(P_{\mathrm{O}_{2}}\right)$ on temperature is displayed. Figures 4(b) and 4(d) represent the theoretical equilibrium phase boundaries between $\mathrm{Ti}_{3} \mathrm{O}_{5}-\mathrm{Ti}_{4} \mathrm{O}_{7}$ and $\mathrm{Ti}_{4} \mathrm{O}_{7}-\mathrm{Ti}_{5} \mathrm{O}_{9}$. The predicted phase boundaries compare very well with those observed, which were taken from Waldner and Eriksson, ${ }^{1}$ and are portrayed in Figs. 4(a) and 4(c). The line labeled as limit in Figs. 4(b) and 4(d) represents the oxygen chemical potential's lower limit-calculated using Eq. (8) - and expressed as a function of the oxygen partial pressure and temperature. That is why the region below that line is labeled as forbidden region. To complement Fig. 4, Table I shows the theoretical and experimental equilibrium temperatures for an oxygen partial pressure of 1 $\times 10^{-15} \mathrm{~atm}$. The agreement between the predicted temperatures and those observed is very good.

\section{DISCUSSION}

Hoshino et $a l .{ }^{49}$ measured the tracer diffusion of titanium as a function of temperature and oxygen partial pressure, and proposed that titanium diffused by an interstitial-type mechanism. They fitted the observed diffusion data with a defect model proposed by Blumenthal and Whitmore ${ }^{50}$ and found reasonable values for the electron mobility and the diffusivity of the interstitial titanium atoms.

More recently, Bak et al. ${ }^{16}$ derived experimental defect concentrations for $\mathrm{TiO}_{2}$ within a wide range of oxygen partial pressures. These defects diagrams indicated that undoped $\mathrm{TiO}_{2}$ exhibits $n$-type properties within the entire range of $p_{\mathrm{O}_{2}}$, which is divided in two regimes. Under extremely reducing conditions $\left(T \approx 1423 \mathrm{~K}\right.$ and $p_{\mathrm{O}_{2}} \leqslant 10^{-16}$ atm), trivalent titaniums are the predominant defects, while oxygen vacancies prevail in the intermediate regime $(T \approx 1423 \mathrm{~K}$ and $1 \times 10^{-8}$ atm $\left.\leqslant p_{\mathrm{O}_{2}} \leqslant 1 \times 10^{-16} \mathrm{~atm}\right)$.

The point labeled as $\mathrm{Ti}_{\text {int }}-\mathrm{O}_{\text {vac }}$ in Fig. 3 indicates that the formation energies of the oxygen vacancy and titanium interstitial are the same. As for the Magnéli phases, it is possible to calculate the relationship between $p_{\mathrm{O}_{2}}$ and $T$ at this point. In particular, at $T=1423 \mathrm{~K}$, the model predicts an oxygen partial pressure of the order of $1 \times 10^{-13} \mathrm{~atm}$. Therefore, for $\mathrm{p}_{\mathrm{O}_{2}} \leqslant 1 \times 10^{-13}$ atm titanium interstitials have a lower formation energy than oxygen vacancies, which is the tendency observed experimentally. However, at that equilibrium point, none of these isolated defects are stable with respect to ideal nondefective rutile and oxygen gas. Their formation energies are, albeit smaller than $0.05 \mathrm{eV}$ per $\mathrm{TiO}_{2}$ unit, still positive. This changes at lower oxygen chemical potentials, where titanium interstitials become stable. In fact, as can be seen in Fig. 2(a), at defect concentrations for which only isolated defects form, titanium interstitials are the ones with the lowest formation energy. This prediction agrees with the observations of Bak et al. ${ }^{16}$

Although this work is concerned specifically with static calculations, i.e., molecular dynamics are not considered, the information obtained can illuminate the observed defect kinetics. Nowotny et al. ${ }^{17}$ studied the kinetics using electrical conductivity measurements in the range 1073-1323 K and $p_{\mathrm{O}_{2}}$ range $1 \times 10^{-17}$ atm to $1 \mathrm{~atm}$. These data were used to determine the chemical diffusion coefficient, which was considered solely in terms of the transport of oxygen vacancies. They found a complex dependence of the coefficient with $p_{\mathrm{O}_{2}}$. In particular, their results show a weak dependence of this diffusion coefficient on $p_{\mathrm{O}_{2}}$ at low oxygen chemical potentials, i.e., in the extremely reduced regime. This result was interpreted as an indication of the defects forming ordered structures. This observation is in agreement with the data presented in Fig. 2(b), which indicates that oxygen defects, at low oxygen chemical potentials, prefer form shear structures, i.e., the Magnéli phases.

It is also possible to speculate about the dynamical processes by which the phases form. The predicted thermodynamics suggests that at low oxygen chemical potential, the titanium interstitial is the preferred isolated point defect, and that any oxygen vacancy defects will aggregate into spatially ordered arrays. This combination provides insight into the 


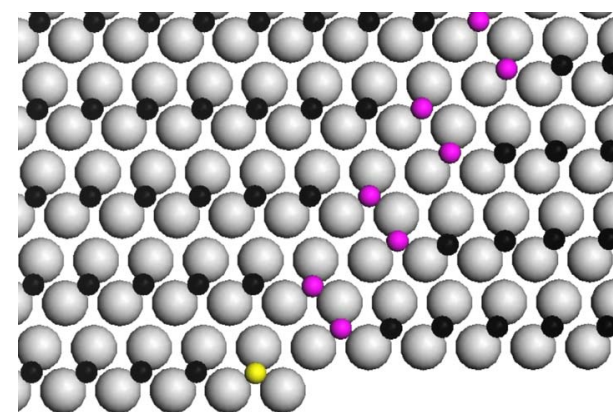

(a)

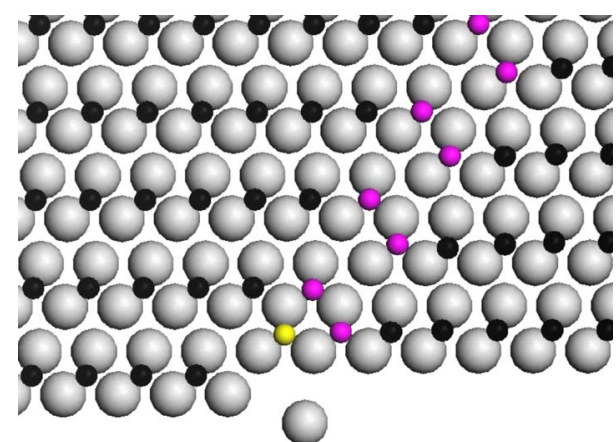

(b)

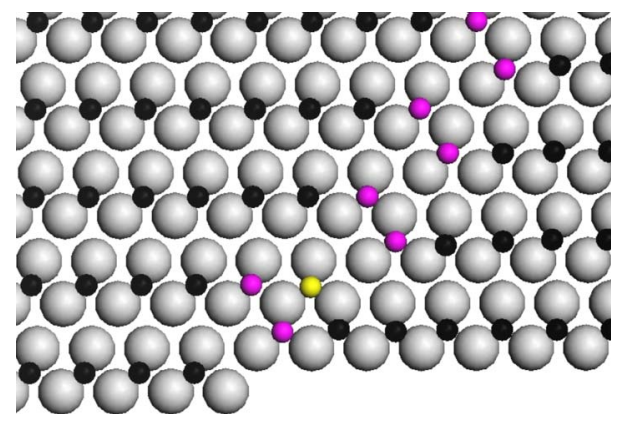

(c)

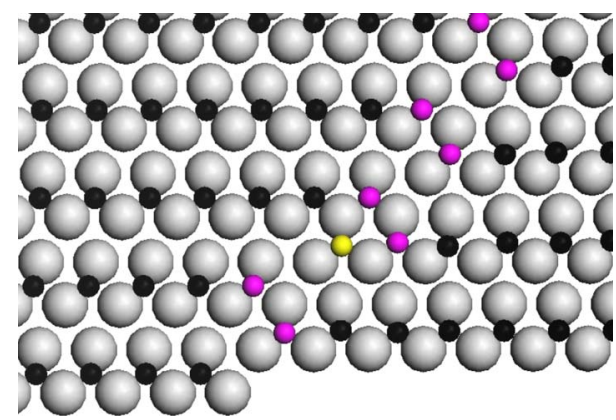

(d)

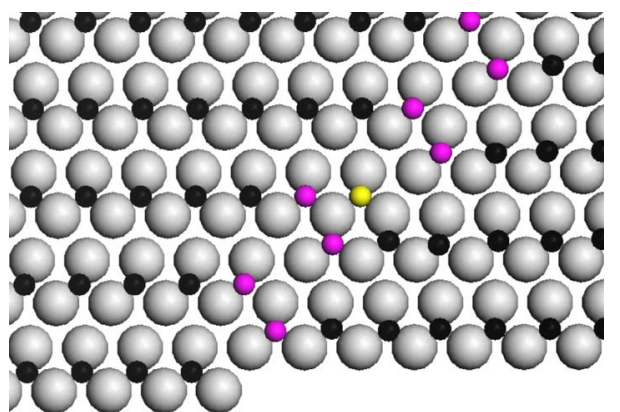

(e)

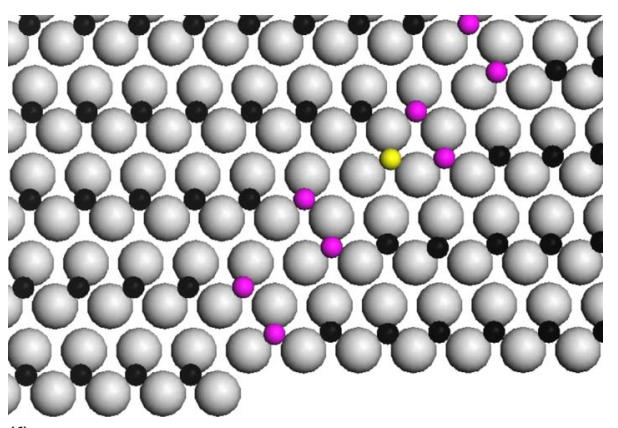

(f)

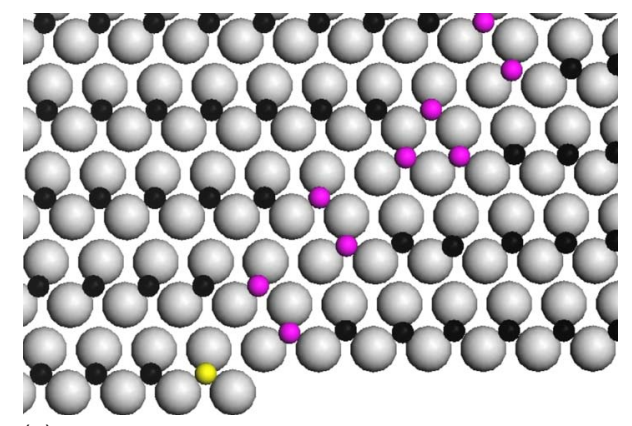

(g)

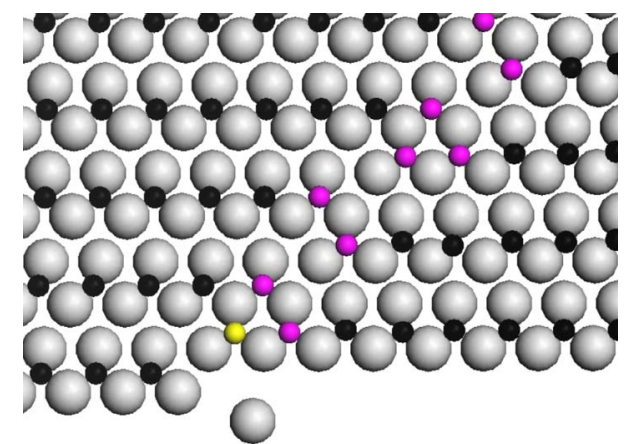

(h)
FIG. 5. (Color online) Panels (a)-(1) show the sequence, on a model (110) cation+anion layer, of the Andersson-Wadsley mechanism for introducing the $\mathrm{CS}$ planes into rutile. mechanisms that have been proposed for the formation of the Magnéli phases.

Andersson and Waldsey ${ }^{51}$ proposed a mechanism for the production of CS planes in rutile which involved migration of titanium atoms. In the reduced regime, the oxygen chemical potential at the surface has a lower value than in the bulk. For a system near or at equilibrium, this implies that the titanium chemical potential is enhanced at the surface [see Eq. (6)]. Therefore, the titanium chemical potential's gradi- ent will point toward the interior of the crystal, favouring the diffusion of titaniums atoms in that direction. The proposed diffusion mechanism involves titanium atoms jumping from an octahedral interstice, normally occupied by cations in rutile, to a neighboring empty octahedral site. The shortest "jump" is specified by the vector $\frac{1}{2}\langle 011\rangle$. As titaniums diffuse away from the surface, they leave nonbonded oxygens behind, which detach from the surface and enter the gas phase. Figures 5(a)-5(1) show the process schematically. 


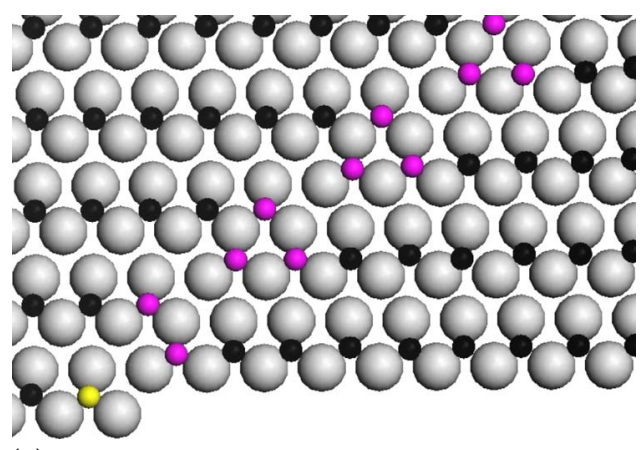

(a)

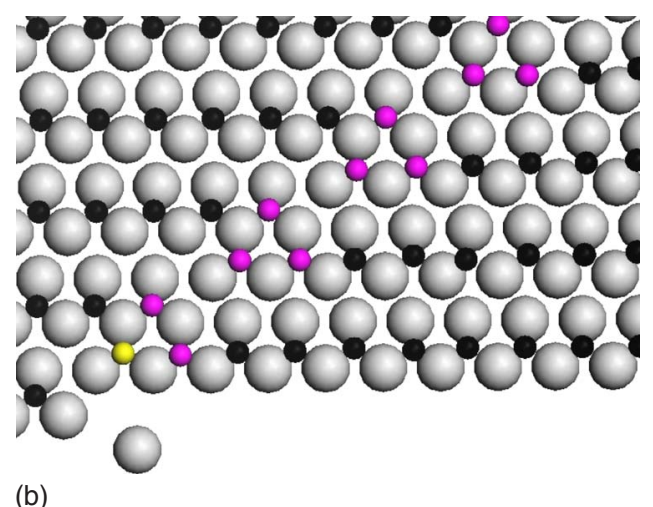

FIG. 6. (Color online) Panels (a) and (b) show the final stages for the Andersson-Wadsley mechanism on a model (110) cation + anion layer. The newly created $Z$-shaped antiphase boundaries are highlighted in purple.

Figure 5(a) shows the cation+anion (100) layer in pure rutile. Highlighted in purple are titanium atoms belonging to an antiphase boundary. These atoms can be thought of as belonging to a dislocation that extends up to the surface. One "normal" titanium is highlighted in yellow. In Fig. 5(b) this titanium has moved to an interstitial position, and one newly created nonbonded oxygen has evaporated from the surface into the gas phase. The first $Z$-shaped antiphase boundary (see Sec. III) appears. Figures 5(c) and 5(d) shows another pure titanium jumping into an interstitial position. The $Z$-shaped antiphase boundary is displaced toward the bulk's interior. Figures 5(e) and 5(f) show a similar process. Figure $5(\mathrm{~g})$ shows a new titanium, close to the surface, migrating into the bulk. Its final position is highlighted in yellow and the unbonded oxygens can be seen leaving the surface. Figure 5(h) shows another internal titanium highlighted in yellow in its final position. It has just jumped into an interstitial position and displaced another $Z$-shaped antiphase boundary.

Figures 6(a) and 6(b) show the process in its final stages. Basically, within this mechanism, the antiphase boundaries are acting as a high conductivity path for transporting titaniums, but without any long-range diffusion. This mechanism requires the formation and mobility of titanium interstitials which, according to our results, are the thermodynamically favored defects at low oxygen chemical potentials.

\section{SPIN}

Spin and its coupling to form magnetic phases in reduced rutile is of great interest $\mathrm{t}^{52}$ but this has only a marginal effect on the structural thermodynamics considered here.

For example, Lindan et al..$^{53}$ performed spin polarized calculations in oxygen-defective bulk rutile with a formalism similar to that used in the current study.

They found that the spin lowered the defect's formation energy by around $0.5 \mathrm{eV}$ which, in our formalism, implies a lowering of the order of $0.01 \mathrm{eV}$ in the normalized formation energy (i.e., per formula unit of the supercell). Figure 3 indicates that such a small contribution can be considered negligible.

For the Magnéli phases, spin polarized calculations performed by the authors confirmed that spin polarization lowers the normalized defect formation energies marginally. For $\mathrm{Ti}_{4} \mathrm{O}_{7}$ and $\mathrm{Ti}_{5} \mathrm{O}_{9}$ the lowering is of the order of $0.06 \mathrm{eV}$, which did not affect the stability ordering of these Magnéli phases. Moreover, the relationship between the oxygen partial pressure and temperature at the $\mathrm{Ti}_{4} \mathrm{O}_{7}$ and $\mathrm{Ti}_{5} \mathrm{O}_{9}$ equilibrium point did not differ significantly from Fig. 4(d).

In summary, although spin is important when studying rutile's defective structures-especially if the electronic structure is being considered-it does not affect the structural thermodynamics significantly.

\section{CONCLUSIONS}

The thermodynamics of rutile's higher oxides has been investigated by means of first principles calculations and compared to available experimental data. This comparison shows that first principles thermodynamics reproduce the experimental observations reasonably well. Atomic relaxations around the oxygen vacancy were computed, and the values were in good agreement with previous theoretical calculations. Results show that, at low oxygen chemical potentials, rutile's oxygen vacancies prefer to be ordered in planes, forming the so called Magnéli phases. But, when the defect concentration is small, at low oxygen chemical potential, titanium interstitials proved to be the stable point defects. These results support the mechanism proposed by Bursill and $\mathrm{Hyde}^{3}$ to explain the origin of the Magnéli phases.

\section{ACKNOWLEDGMENTS}

We would like to thank Giuseppe Mallia, Keith Refson, and Barbara Montanari for the fruitful discussion of this work. The calculations performed for the isolated defects made use of the facilities of HPCx, the UK's national highperformance computing service, which is provided by EPCC at the University of Edinburgh and by CCLRC Daresbury Laboratory, and funded by the Office of Science and Technology through EPSRC's High End Computing Programme. Calculations on the Magnéli phases were performed on the computing resources provided by STFC's e-Science facility. This work was funded by the EPSRC under the multiscale modeling approach to engineering functional coatings' initiative (EP/C524322/1). 
*1.liborio@imperial.ac.uk

${ }^{1}$ P. Waldner and G. Eriksson, CALPHAD: Comput. Coupling Phase Diagrams Thermochem. 23, 189 (1997).

${ }^{2}$ G. J. Wood and L. A. Bursill, Proc. R. Soc. London, Ser. A 375, 105 (1981).

${ }^{3}$ L. A. Bursill and B. G. Hyde, Prog. Solid State Chem. 7, 177 (1972).

${ }^{4}$ I. Dawson, P. W. Bristowe, J. A. White, and M. C. Payne, in Solid-State Chemistry of Inorganic Materials, edited by P. K. Davies, A. J. Jacobson, C. C. Torardi, and T. A. Vanderah, MRS Symposia Proceedings No. 453 (Materials Research Society, Pittsburgh, 1997), p. 203.

${ }^{5}$ M. M. Islam, T. Bredow, and A. Gerson, Phys. Rev. B 76, 045217 (2007).

${ }^{6}$ E. Cho, S. Han, H. S. Ahn, K. R. Lee, S. K. Kim, and C. S. Hwang, Phys. Rev. B 73, 193202 (2006).

${ }^{7}$ S. Andersson and L. Jahnberg, Ark. Kemi 21, 413 (1963).

${ }^{8}$ K. Yoshida, T. Kawai, T. Nambara, S. Tanemura, K. Saitoh, and N. Tanaka, Nanotechnology 17, 3944 (2006).

${ }^{9}$ J. Yahia, Phys. Rev. 130, 1711 (1963).

${ }^{10}$ P. Kofstad, J. Less-Common Met. 13, 635 (1967).

${ }^{11}$ R. N. Blumenthal, J. Coburn, J. Baukus, and W. M. Hirthe, J. Phys. Chem. Solids 27, 643 (1966).

${ }^{12}$ J. He and S. Sinnott, J. Am. Ceram. Soc. 88, 737 (2005).

${ }^{13}$ M. K. Nowotny, T. Bak, J. Nowotny, and C. C. Sorrell, Phys. Status Solidi A 242, R88 (2005).

${ }^{14}$ M. K. Nowotny, T. Bak, and J. Nowotny, J. Phys. Chem. B 110, 16270 (2006).

${ }^{15}$ M. K. Nowotny, T. Bak, and J. Nowotny, J. Phys. Chem. B 110, 21560 (2006)

${ }^{16}$ T. Bak, J. Nowotny, M. Rekas, and C. C. Sorrell, J. Phys. Chem. Solids 64, 1057 (2003).

${ }^{17}$ M. K. Nowotny, T. Bak, and J. Nowotny, J. Phys. Chem. B 110, 16292 (2006)

${ }^{18}$ A. Fujishima and K. Honda, Nature (London) 238, 37 (1972).

${ }^{19}$ U. Diebold, Surf. Sci. Rep. 48, 53 (2003).

${ }^{20}$ J. R. Smith, F. C. Walsh, and R. L. Clarke, J. Appl. Electrochem. 28, 1021 (1998).

${ }^{21}$ O. Storz, H. Gasthuber, and M. Woydt, Surf. Coat. Technol. 140, 76 (2001).

${ }^{22}$ R. F. Bartholomew and D. R. Frankl, Phys. Rev. 187, 828 (1969).

${ }^{23}$ A. D. Inglis, Y. L. Page, P. Strobel, and C. M. Hurd, J. Phys. C 16, 317 (1983)

${ }^{24}$ M. Marezio, D. B. Mc. Whan, P. D. Dernier, and J. P. Remeika, Phys. Rev. Lett. 28, 1390 (1972).

${ }^{25}$ M. Abbate, R. Potze, G. A. Sawatzky, C. Schlenker, H. J. Lin, L. H. Tjeng, C. T. Chen, D. Teehan, and T. S. Turner, Phys. Rev. B 51, 10150 (1995).

${ }^{26}$ S. Lakkis, C. Schlenker, B. K. Chakraverty, and R. Buder, Phys.
Rev. B 14, 1429 (1976).

${ }^{27}$ K. Kobashi, T. Susaki, A. Fujimori, T. Tongai, and H. Takagi, Europhys. Lett. 59, 868 (2002).

${ }^{28}$ M. Watanabe, W. Ueno, and T. Hayashi, J. Lumin. 122-123, 393 (2007).

${ }^{29}$ I. Leonov, A. N. Yaresko, V. N. Antonov, U. Schwingenschlogl, and V. Eyert, J. Phys.: Condens. Matter 18, 10955 (2006).

${ }^{30}$ L. N. Mulay and W. J. Danley, J. Appl. Phys. 41, 877 (1970).

${ }^{31}$ L. K. Keys and L. N. Mulay, Phys. Rev. 154, 453 (1967).

${ }^{32}$ K. Johnston, M. R. Castell, A. T. Paxton, and M. W. Finnis, Phys. Rev. B 70, 085415 (2004).

${ }^{33}$ K. Reuter and M. Scheffler, Phys. Rev. B 65, 035406 (2001).

${ }^{34}$ L. Liborio, Ph.D. thesis, Queen's University Belfast, 2005.

${ }^{35}$ G. X. Qian, R. M. Martin, and D. J. Chadi, Phys. Rev. Lett. 60, 1962 (1988).

${ }^{36}$ N. Esser, A. I. Shkrebtii, U. Resch-Esser, C. Springer, W. Richter, W. G. Schmidt, F. Bechstedt, and R. D. Sole, Phys. Rev. Lett. 77, 4402 (1996).

${ }^{37}$ C. V. de Walle and J. Neugebauer, J. Appl. Phys. 95, 3851 (2004).

${ }^{38}$ M. W. Chase, C. Davies, J. R. Downey, D. J. Frurip, R. A. Mcdonald, and A. N. Syverud, J. Phys. Chem. Ref. Data Suppl. 14, 1 (1985).

${ }^{39}$ S. J. Clark, M. D. Seagall, C. J. Pickard, P. J. Hasnip, M. J. Probert, K. Refson, and M. C. Payne, Z. Kristallogr. 220, 567 (2005).

${ }^{40}$ D. Vanderbilt, Phys. Rev. B 41, 7892 (1990).

${ }^{41}$ P. F. Chester, J. Appl. Phys., Suppl. 32, 2233 (1961).

${ }^{42}$ J. K. Burdett, T. Hughbanks, G. J. Miller, J. W. Richardson, and J. V. Smith, J. Am. Chem. Soc. 109, 3639 (1987).

${ }^{43}$ S. Asbrink and A. Magneli, Acta Crystallogr. 12, 575 (1959).

${ }^{44}$ P. J. Kofstad, J. Phys. Chem. Solids 23, 1579 (1962).

45 J. F. Marucco, J. Gautron, and P. Lemasson, J. Phys. Chem. Solids 42, 363 (1981).

${ }^{46}$ G. Levin and C. J. Rosa, Z. Metallkd. 70, 646 (1979).

${ }^{47}$ C. R. A. Catlow and R. James, Proc. R. Soc. London, Ser. A 384, 157 (1982)

${ }^{48}$ J. S. Anderson and A. S. Khan, J. Less-Common Met. 22, 219 (1970).

${ }^{49}$ K. Hoshino, N. L. Paterson, and C. L. Wiley, J. Phys. Chem. Solids 46, 1397 (1985).

${ }^{50}$ R. N. Blumenthal and D. H. Whitmore, J. Electrochem. Soc. 110, 92 (1963).

${ }^{51} \mathrm{~S}$. Andersson and A. D. Waldsey, Nature (London) 211, 581 (1966).

${ }^{52}$ M. Schmidt, W. Ratcliff, P. G. Radaelli, K. Refson, N. M. Harrison, and S. W. Cheong, Phys. Rev. Lett. 92, 056402 (2004).

${ }^{53}$ P. J. D. Lindan, N. M. Harrison, M. J. Gillan, and J. A. White, Phys. Rev. B 55, 15919 (1997). 